\title{
Preoperative Chemoradiotherapy with Capecitabine and Oxaliplatin in Locally Advanced Rectal Cancer. A Phase I-II Multicenter Study of the Dutch Colorectal Cancer Group
}

\author{
Geke A. Hospers, ${ }^{1}$ Cornelis J. A. Punt, ${ }^{2}$ Margot E. Tesselaar, ${ }^{3}$ Annemieke Cats, ${ }^{4}$ \\ Klaas Havenga, ${ }^{5}$ Jan W. H. Leer, ${ }^{6}$ Corrie A. Marijnen, ${ }^{3}$ Edwin P. Jansen, ${ }^{7}$ \\ Han H. J. M. Van Krieken, ${ }^{8}$ Theo Wiggers, ${ }^{5}$ Cornelis J. H. Van de Velde, ${ }^{9}$ \\ and Nanno H. Mulder ${ }^{1}$
}

\footnotetext{
${ }^{1}$ Medical Oncology, University Medical Center Groningen and University of Groningen, Groningen, The Netherlands

${ }^{2}$ Medical Oncology, St. Radboud University Medical Center, Nijmegen, The Netherlands

${ }^{3}$ Clinical Oncology, Leiden University Medical Center, Leiden, The Netherlands

${ }^{4}$ Gastroenterology, Netherlands Cancer Institute, Amsterdam, The Netherlands

${ }^{5}$ Surgical Oncology, University Medical Center Groningen and University of Groningen, Groningen, The Netherlands

${ }^{6}$ Radiotherapy, St. Radboud University Medical Center, Nijmegen, The Netherlands

${ }^{7}$ Radiotherapy, Netherlands Cancer Institute, Amsterdam, The Netherlands

${ }^{8}$ Pathology, St. Radboud University Medical Center, Nijmegen, The Netherlands

${ }^{9}$ Surgical Oncology, Leiden University Medical Center, Leiden, The Netherlands
}

\begin{abstract}
Background: We studied the maximum tolerated dose (MTD) and efficacy of oxaliplatin added to capecitabine and radiotherapy (Capox-RT) as neoadjuvant therapy for rectal cancer.

Methods: T3-4 rectal cancer patients received escalating doses of oxaliplatin (day 1 and 29) with a fixed dose of capecitabine of $1000 \mathrm{mg} / \mathrm{m}^{2}$ twice daily (days $1-14,25-38$ ) added to RT with 50.4 Gy and surgery after 6-8 weeks. The MTD, determined during phase I, was used in the subsequent phase II, in which $\mathrm{R} 0$ resection rate (a negative circumferential resection margin) was the primary end point.

Results: Twenty-one patients were evaluable. In the phase I part, oxaliplatin at $85 \mathrm{mg} / \mathrm{m}^{2}$ was established as MTD. In phase II, the main toxicity was grade III diarrhea (18\%). All patients underwent surgery, and 20 patients had a resectable tumor. An R0 was achieved in $17 / 21$ patients, downstaging to T0-2 in $7 / 21$ and a pCR in $2 / 21$.

Conclusion: Combination of Capox-RT has an acceptable acute toxicity profile and a high $\mathrm{R} 0$ resection rate of $81 \%$ in locally advanced rectal cancer. However the pCR rate was low.

Key Words: Rectal cancer-Radiotherapy-Oxaliplatin-Capecitabine-ChemoradiationPhase I-II study.
\end{abstract}

Colorectal cancer is a major public health problem in the Western world and ranks as the third leading

Received December 8, 2006; accepted February 7, 2007; published online: July 26, 2007.

Address correspondence and reprint requests to: Geke A. Hospers; E-mail: g.a.p.hospers@int.umcg.nl

Published by Springer Science+Business Media, LLC $\odot 2007$ The Society of Surgical Oncology, Inc. cause of death in both males and females. In 2000, more than 9000 new colorectal cancer patients were registered in the Netherlands, of whom 25\% had rectal cancer. ${ }^{1}$ Surgical resection is the only curative treatment. However, following potentially curative resection, local recurrence rate varies between 5 and $40 \% .^{2-4}$ Total mesorectal excision (TME) is now the standard technique for primary resectable rectal 
cancer and has significantly improved local control. ${ }^{4-6}$ The cornerstone of the TME technique is the complete removal of the rectum and mesorectum to realize free circumferential resection margins. Adam showed in 1994 that the incidence of local recurrence 5 years after resection will rise from $10-78 \%$ in case of circumferential margin (CRM) involvement. ${ }^{7}$ So downstaging before the TME procedure may decrease the incidence of CRM involvement and local recurrence. In the Dutch TME trial, no tumor downstaging was detected in the week after $5 \times 5 \mathrm{~Gy} .{ }^{8}$ A recent Polish trial demonstrated that a radiotherapy schedule of 50.4 Gy combined with chemotherapy (5-FU/Leucovorin) followed after 4-6 weeks by surgery resulted in a significant higher percentage of downstaging compared with short-term preoperative radiotherapy of $5 \times 5$ Gy followed by surgery within 7 days. ${ }^{9}$ So downstaging is dependent on both the total radiotherapy dose and the interval between the end of the radiotherapy and the surgery. In general, to achieve downstaging this interval needs to be at least 6 weeks and the dose needs to be at least $45 \mathrm{~Gy} .{ }^{9}$ Although the obtained downstaging after neoadjuvant radiochemotherapy did result in a favorable prognosis, ${ }^{10}$ it was not clear until recently whether the addition of chemotherapy to preoperative radiotherapy could increase the amount of downstaging and thus improve local control. The evidence that the addition of chemotherapy to preoperative radiotherapy improves local control rates has recently been shown by two separate trials. The EORTC 22921 trial has a two by two factorial design and randomized between preoperative radiotherapy (45 Gy) versus preoperative chemoradiotherapy (45 Gy combined with 5-FU/Leucovorin). The results demonstrated an increased local control rate for the chemoradiation arm: $91 \%$ versus $83 \% .^{11,12} \mathrm{~A}$ similar result was found in the French FFCD 9203 study, which randomized between preoperative radiotherapy (45 Gy) and preoperative chemoradiotherapy (45 Gy and 5-FU/ Leucovorin) and which showed local recurrence rates of $16.5 \%$ and $8 \%$, respectively. ${ }^{13}$ Based on these studies, prolonged preoperative chemoradiation is considered the standard treatment for resectable locally advanced rectal cancers. The EORTC study and the French study employed 5FU bolus injection modulated with leucovorin. However 5FU administered by continuous infusion or orally (capecitabine/ UFT) may be more effective and less toxic than 5FU administered by bolus injection. ${ }^{14-16}$ In parallel, the results of palliative chemotherapy in advanced colorectal cancer have been substantially improved by the combination of $5 \mathrm{FU}$ with oxaliplatin or irinotecan (reviewed by Punt $^{17}$ ). Both in vivo and in vitro, oxaliplatin has been shown to have at least an additive interaction with radiotherapy in the management of digestive tract tumors. ${ }^{18-20}$ Incorporation of those drugs in combined treatment strategies could substantially improve the results obtained with bolus $5 \mathrm{FU}$ alone in downstaging and $\mathrm{R} 0$ resection rates in patients with locally advanced rectal cancer.

We investigated the combination of capecitabine and oxaliplatin given concomitantly with radiotherapy in patients with locally advanced rectal cancer. The optimal dose of oxaliplatin was first established in a phase I study, and in the following phase II study the efficacy of this regimen was tested.

\section{PATIENTS AND METHODS}

\section{Objectives}

The objective of the phase I study was to determine the maximum tolerated dose (MTD) of oxaliplatin in combination with a fixed dose of capecitabine and radiotherapy, and the objective of the phase II study was to determine the R0 resection rate and pathological complete response rate (pCR).

\section{Eligibility Criteria}

Eligibility criteria included histologically documented adenocarcinoma of the rectum within $15 \mathrm{~cm}$ from the anal sphincter, locally advanced stage T3 (distance to the endopelvic fascia $<2 \mathrm{~mm}$ ) or T4 tumors based on computed tomography (CT) or magnetic resonance imaging (MRI) findings, performance status (Eastern Cooperative Oncology Group) 0-2, age $>18$ years old, adequate hematological, liver function, and other laboratory parameters (white blood cells $3.0 \times 10^{9} / \mathrm{L}$, platelets $>100 \times 10^{9} / \mathrm{L}$, creatinin clearance $>50 \mathrm{~mL} / \mathrm{min}$, bilirubin $<1.5$ times the upper limit of the normal range (ULN), and written informed consent. Patients with reproductive potential should use adequate contraceptive measures. Patients were excluded in case of prior chemotherapy and/or pelvic radiotherapy, acute bowel obstruction without colostomy, uncontrolled ischemic heart disease, peripheral neuropathy, or any uncontrolled serious systemic disease. The protocol was approved by the local ethics committees of all four participating hospitals. The study was performed within the framework of the Dutch Colorectal Cancer Group (DCCG). 


\section{TREATMENT}

\section{Radiotherapy}

All patients received radiation delivered by an isocentric three- or four-field technique, using a linear accelerator of at least $10 \mathrm{MV}$. The patients were treated in either supine or prone position with a full bladder. The radiation field extended superiorly to the L5/S1 junction and covered inferiorly the obturator foramina. The minimal inferior border extended $4-5 \mathrm{~cm}$ below the tumor. In case the tumor was located in the lower third of the rectum, the perineum was encompassed in the treatment field. The width of the AP-PA portals had to cover the lateral pelvic inlet with a margin of $1.5 \mathrm{~cm}$. The entire sacrum was included with a dorsal margin of $1.5 \mathrm{~cm}$. Anteriorly, the lateral fields had to encompass the tumor as determined by barium enema (optional) and pelvic CT scan. If there was clinical evidence of involvement of the bladder, the prostate, the cervix or the uterine body, not only the internal iliac nodes but also the external iliac nodes were included in the radiation field. Computerized dosimetry was routinely performed. Irradiation was delivered 5 days per week at a dose of $1.8 \mathrm{~Gy} /$ day to a total dose of $45 \mathrm{~Gy}$ with a boost to the tumor in 3 fractions of $1.8 \mathrm{~Gy}$ to a total of $50.4 \mathrm{~Gy}$.

\section{Chemotherapy}

Capecitabine was administered orally twice daily at $1000 \mathrm{mg} / \mathrm{m}^{2}$ on days $1-14$ and $25-38$.

Oxaliplatin was given twice by intravenous infusion over $2 \mathrm{~h}$, on the first day of radiotherapy and on day 29. Oxaliplatin doses were planned at 85, 100, and $135 \mathrm{mg} / \mathrm{m}^{2}$. Each dose level was to be administered to at least three patients. If a dose-limiting toxicity (DLT) was observed in $\geq 1$ patient, a total of 6 patients had to be treated at that dose level. The maximum-tolerated dose (MTD) was defined as the level at which $\leq 2$ out of 6 patients experienced DLTs without compromizing radiotherapy and surgery and was the recommended dose in the phase II study.

\section{Surgery}

Surgical resection was performed 6-8 weeks after completion of the chemoradiation therapy. The resection was performed according to the principles of total mesorectal excision as described by $\mathrm{Heald}^{2}$ and Enker. ${ }^{21}$ These principles include sharp dissection under direct vision following the lipoma-like surface of the mesorectum. Proximal transection of the inferior mesenteric artery was in general performed distal to the collateral between the left colic artery and branches of the sigmoid artery. Anterior dissection in male patients was carried out in front of Denonvilliers' fascia. If tumor extended into the prostate a total exenteration was performed. In female patients, resection of the posterior vaginal wall was performed if necessary. A distal mesorectal margin of $2 \mathrm{~cm}$ was considered adequate if bowel continuity was to be restored.

\section{Pathology}

Pathology evaluation was standardized according to national guideliness. The CRM was assessed according to the method of Quirke et al., ${ }^{7}$ and a margin of $<1 \mathrm{~mm}$ from the primary tumor to the endopelvic fascia is considered positive.

\section{Statistics}

Twenty patients were to be included at the MTD to detect with a power of $80 \%$ a $\mathrm{R} 0$ resectability rate of $>80 \%$ (with a $95 \%$ CI of $56-94 \%$ ).

\section{Monitoring and Management During Treatment}

Physical examination and evaluation of toxicity was performed weekly. Complete blood count, electrolytes, creatinine, and total protein were determined weekly. Standard antiemetic prophylaxis with 5HT3 antagonist prior to oxaliplatin infusion was used. Adverse events were classified according to the National Cancer Institute (NCI) Common Toxicity Criteria (CTC) Version 2.0. Global quality of life (QOL) assessment was evaluated weekly during the chemoradiotherapy using a visual analogue scale (VASQOL) in which in a single $10 \mathrm{~cm}$ line anchored on the left with "worst" and on the right with "best" for QOL. ${ }^{22}$ Dose-limiting toxicities were defined as white blood cell (WBC) and platelets $<3.0$ and $<100 \times 10^{9} / \mathrm{L}$, respectively, before start of the next treatment cycle with oxaliplatin after a maximum delay of one week; diarrhea grade $>2$; oral mucositis grade $>1$; skin toxicity grade $>2$ before start of next treatment cycle with capecitabine, despite a delay of dosing for 1 week; neurosensory toxicity grade $>2$ despite a delay in dosing for 1 week; occurrence of any grade 4 toxicity; and any event that would compromise the administration of radiotherapy. 


\section{RESULTS}

\section{Patients Characteristics}

Between June 2003 and December 2004, 22 patients with T3/T4 rectal cancer from four different centers were enrolled in the study. Patient characteristics are shown in Table 1. All patients were evaluable for toxicity during chemoradiation. One patient was lost to follow-up after chemoradiation (surgery was performed abroad). Therefore, 21 patients were evaluable for clinical outcome after surgery. Twenty patients $(91 \%)$ received the planned dose of chemotherapy. All patients received the planned dose of radiotherapy. The median follow-up was 14 months.

\section{Toxicity}

At the first oxaliplatin dose level of $85 \mathrm{mg} / \mathrm{m}^{2}$, one patient experienced DLT (hospitalization for grade 3 nausea and grade 2 diarrhea). This dose level was then expanded to six patients, and DLT was observed in another patient (hospitalization for grade 3 diarrhea). Therefore, a dose of $85 \mathrm{mg} / \mathrm{m}^{2}$ oxaliplatin was considered the MTD. In the additional 16 patients, two more patients experienced grade 3 diarrhea. Hand-foot syndrome, myelosuppression, and neurotoxicity were observed only in a minority of patients and were of mild severity. The grade $3 / 4$ toxicity data are shown in Table 2.

\section{Surgery}

TME surgery was performed after a median of 47 days (range 4-8 weeks range in days) following the completion of chemoradiation. Mean hospitalization time was 14 days (range 9-95; 70\% < 21 days). The main surgical complications were: major bleeding (1), rectal perforation (1), ureter lesion (1). Postsurgery treatment related complications were seen in 12 patients with mild wound infections (6), wound dehiscences (2), (sub)ileus (4), rhabdomyolysis (1), and in one patient a life-threatening multiorgan failure after a perforation in an abcess located near the anus praeter. Rhabdomyolysis is an unusual complication. In this patient it was probably caused by the operation procedure and not by this specific procedure as there were no other postoperative complications. Three patients (15\%) needed reoperation for perforation (1) and pelvic abscesses (2). The 60-day mortality rate was nil.
TABLE 1. Patient characteristics $(n=22)$

\begin{tabular}{ll}
\hline Characteristics & No. \\
\hline Median age (years) & 58 \\
Range (years) & $45-70$ \\
Men/Women & $12 / 10$ \\
Performance status & 3 \\
0 & 19 \\
1 & \\
Distance of tumor to anal verge $(\mathrm{cm})$ & 11 \\
$0-5$ & 7 \\
$5-10$ & 3 \\
$10-15$ & 1 \\
Unknown & 17 \\
$T$ status & 5 \\
T3 ${ }^{a}$ & \\
T4 ${ }^{b}$ & \\
\hline${ }^{a}$ T3 beyond the fascia recti. & \\
${ }^{b}$ T4: into pelvic organs $(1$ ureter, 2 vagina, 2 prostate) & \\
&
\end{tabular}

\section{Efficacy}

Twenty-one patients with a T3-T4 tumor based on CT or MRI underwent surgery with 10 abdominoperineal resections (APRs) and 10 low anterior resections (LARs); in one patient the tumor was not resectable. A R0 resection was achieved in 17 patients (81\%, 95\% CI 58-95\%). A pCR was observed in 2 patients $(10 \%, 95 \%$ CI $1-30 \%)$, and in one patient (5\%) the surgical specimen only showed minimal microscopic disease. The postradiotherapeutic pathologic staging yielded in seven patients $(33 \%$; $95 \% \mathrm{CI}$ 15-57\%) a downstaging to pT0-T2. After a median follow-up of 14 months, four patients have died due to metastatic disease and one patient has experienced a local recurrence.

\section{Quality of Life}

The VASQOL score was measured weekly during chemoradiotherapy. The QOL decreased after 4 weeks treatment from $80 \%$ in week 4 to $60 \%$ in week $6(p<.002$, student-t test $)$.

\section{DISCUSSION}

Based on the EORTC 22921 and FFCD 9203 phase III studies, ${ }^{11,13,23}$ preoperative chemoradiation with 5FU and $45 \mathrm{~Gy}$ or higher is now considered to be the standard of care for locally advanced rectal cancer. In these studies, 5FU was given as a daily bolus infusion in week 1 and 5. To improve on these results, we tested the feasibility and efficacy of the addition of oxaliplatin to capecitabine in this combined treatment strategy. Our recommended dose for 
TABLE 2. Toxicity in the study population $(n=22)$

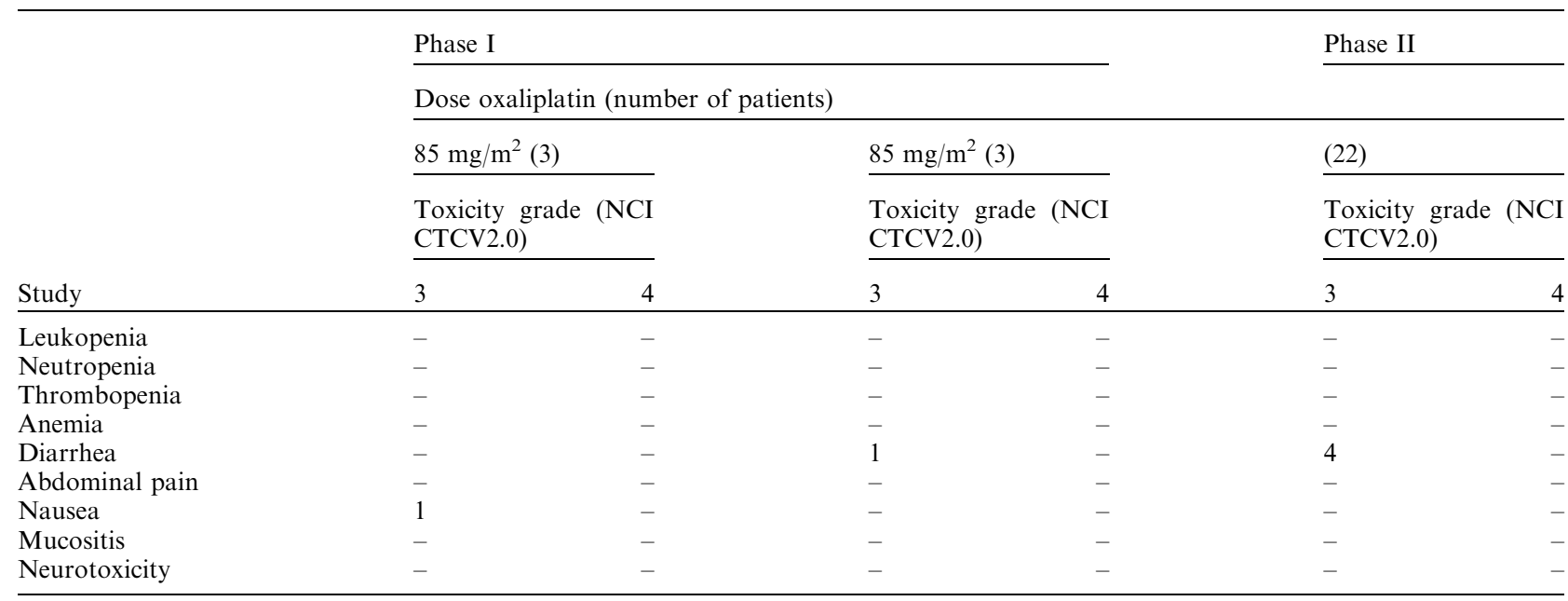

TABLE 3. Locally advanced rectal cancer, recent neoadjuvant studies with fluoropyrimidines and oxaliplatin in combination with radiotherapy

\begin{tabular}{|c|c|c|c|c|c|c|c|c|c|c|}
\hline \multirow[b]{2}{*}{ Study } & \multirow[b]{2}{*}{$\begin{array}{l}\text { Design } \\
\text { (phase) }\end{array}$} & \multirow[b]{2}{*}{ Stage } & \multirow[b]{2}{*}{$N$} & \multirow[b]{2}{*}{$\begin{array}{l}\text { EBRT } \\
(\mathrm{Gy})\end{array}$} & \multirow[b]{2}{*}{ CT } & \multirow[b]{2}{*}{$\begin{array}{l}\mathrm{R} 0 \\
(\%)\end{array}$} & \multirow[b]{2}{*}{$\begin{array}{l}\mathrm{pCR} \\
(\%)\end{array}$} & \multicolumn{3}{|c|}{ Toxicity grade $3-4(\%)$} \\
\hline & & & & & & & & Total & Diarrhea & Myelotoxic \\
\hline \multicolumn{11}{|c|}{ Preoperative radiotherapy vs. chemoradiotherapy (with $5 F U$ ) } \\
\hline \multirow[t]{2}{*}{ EORTC $22921^{11,23}$} & III & $\mathrm{T} 3 / \mathrm{T} 4$ & 1011 & 45 & - & - & 5 & $38^{a}$ & $17-$ & $1-$ \\
\hline & & & & 45 & $5 F U$ pre & - & 14 & 54 & 34 & $9-$ \\
\hline \multirow{2}{*}{ FFCD $9203^{13}$} & III & $\mathrm{T} 3 / \mathrm{T} 4$ & 762 & 45 & - & 90 & 4 & 3 & - & - \\
\hline & & & & 45 & $5 \mathrm{FU}$ pre & 91 & 12 & 15 & - & - \\
\hline \multirow[t]{2}{*}{ Bujko $^{9}$} & III & $\mathrm{T} 3 / \mathrm{T} 4$ & 316 & $5 \times 5$ & - & 87 & 1 & 3 & - & - \\
\hline & & & & 50.4 & $5 \mathrm{FU}$ pre & 96 & 17 & 18 & - & - \\
\hline \multicolumn{11}{|c|}{ Preoperative CRT with capecitabine } \\
\hline $\mathrm{Kim}^{30}$ & II & $\mathrm{T} 3 / \mathrm{T} 4 / \mathrm{N}+$ & 45 & 50.4 & Cap 3 & - & 31 & 17 & 4 & - \\
\hline Dunst $^{31} /$ Glynne- Jones ${ }^{32}$ & II & $\mathrm{T} 3 / \mathrm{T} 4 / \mathrm{N}+$ & 98 & $50.4+5.4$ & Cap 1 & - & 4 & 22 & 4 & 10 \\
\hline $\mathrm{Chau}^{33}$ & II & $\mathrm{T} 3+$, low $\mathrm{T} 3, \mathrm{~N} 2$ & 77 & 54 & Cap $1^{b}$ & 99 & 24 & \multicolumn{3}{|c|}{4 deaths } \\
\hline \multirow[t]{2}{*}{ Yerushalmi ${ }^{16}$} & II & $\mathrm{T} 3 / \mathrm{T} 4(\mathrm{~T} 210 \%)$ & 46 & 50.4 & 5 FU vs Cap 2 & - & 17 & & 4 & \\
\hline & & & 43 & & & - & 30 & & 2 & \\
\hline \multicolumn{11}{|c|}{ Preoperative CRT with $5 \mathrm{FU}$ based chemotherapy and oxaliplatin } \\
\hline Gambacorta ${ }^{34}$ & II & $\mathrm{T} 3$ & 30 & 50 & Tomox & - & 30 & 13 & 3 & 10 \\
\hline Gerard $^{26}$ & II & $\mathrm{T} 2 / \mathrm{T} 3 / \mathrm{T} 4$ & 40 & 50.4 & $\mathrm{FU}+\mathrm{Ox} 1$ & - & 15 & 18 & 5 & 3 \\
\hline Rodel $^{35}$ & II & $\mathrm{T} 3-4$ & 32 & 50.4 & Capox 4 & 79 & 19 & 16 & 8 & 0 \\
\hline Carraro $^{36}$ & II & $\mathrm{T} 3 / \mathrm{T} 4$ & 22 & 50.4 & $\mathrm{FU}+\mathrm{Ox} 2$ & - & 14 & - & 27 & 14 \\
\hline Aschele $^{25}$ & $\mathrm{I} / \mathrm{II}$ & $\mathrm{T} 3 / \mathrm{T} 4 / \mathrm{N}+$ & 25 & 50.4 & $\mathrm{FU}+\mathrm{Ox} 3$ & 89 & 28 & 24 & 16 & 4 \\
\hline Glynne-Jones $^{32}$ & $\mathrm{I} / \mathrm{II}$ & Unresectable & 94 & 45 & Capox 2 & 78 & 28 & 13 & 10 & - \\
\hline Machiels ${ }^{24}$ & II & $\mathrm{T} 3 / \mathrm{T} 4 / \mathrm{N}+$ & 40 & 45 & Capox 1 & 83 & 14 & - & 30 & - \\
\hline Hospers (2006) This study & $\mathrm{I} / \mathrm{II}$ & $\mathrm{T} 3 / \mathrm{T} 4$ & 22 & 50.4 & Capox & 81 & 10 & _- & 18 & _- \\
\hline $\begin{array}{l}\text { Cap 1: } 825 \mathrm{mg} / \mathrm{m}^{2} \\
\quad 2 \times \text { daily, } 7 \text { days }\end{array}$ & \multicolumn{5}{|c|}{$\begin{array}{l}\text { Capox 1: Cap } 8252 \times \text { daily, } \\
\text { Monday-Friday, } \\
\text { oxali } 50 \text { weekly }\end{array}$} & \multicolumn{5}{|c|}{$\begin{array}{l}\text { Tomox: raltitrexed 3, oxali } 130 \text {, } \\
\quad \text { day } 1,19,38\end{array}$} \\
\hline Cap 2: $825 \mathrm{mg} / \mathrm{m}^{2}$ & \multirow{3}{*}{\multicolumn{5}{|c|}{$\begin{array}{l}\text { Capox 2: Cap } 650 \\
2 \times \text { daily, } 7 \text { days, } \\
\text { oxali } 130 \text { per } 2 \text { weeks }\end{array}$}} & \multirow{3}{*}{\multicolumn{5}{|c|}{$\begin{array}{l}\text { FU }+ \text { Ox } 1: 5 \text { FU } 350 \text { daily, } \\
\quad \text { Oxali } 130 \text { weekly, week } 1 \text { and } 5\end{array}$}} \\
\hline $2 \times$ daily, & & & & & & & & & & \\
\hline \multirow{3}{*}{$\begin{array}{l}\text { Cap 3: } 825 \mathrm{mg} / \mathrm{m}^{2} \\
2 \times \text { daily, } \\
14 \text { days }(2 \times)\end{array}$} & \multicolumn{3}{|c|}{ Capox 3: Cap 825} & & & \multirow{2}{*}{\multicolumn{5}{|c|}{$\begin{array}{l}\mathrm{FU}+\mathrm{Ox} 2: 5 \mathrm{FU} 375 / 4 \text { days, } \\
\quad \text { Oxali } 25 / 4 \text { days, week } 1 \text { and } 5\end{array}$}} \\
\hline & \multicolumn{5}{|c|}{$\begin{array}{l}2 \times \text { daily, } 7 \text { days, } \\
\text { oxali } 60 \text { per } 2 \text { weeks }\end{array}$} & & & & & \\
\hline & $\begin{array}{r}\text { Capox } 4 \\
2 \times 14 \\
\text { day } 1\end{array}$ & $\begin{array}{l}\text { 4: Cap } 8252 \times \text { daily, } \\
4 \text { days, oxali } 50 \\
8,22,29\end{array}$ & & & & \multicolumn{5}{|c|}{$\begin{array}{l}\text { FU + Ox 3: 5FU } 225 \text { daily, } \\
\text { Oxali } 60 \text { weekly }\end{array}$} \\
\hline
\end{tabular}

${ }^{a} \geq$ Grade 2 toxicity.

${ }^{b} 12$ weeks neoadjuvant CAPO. 
the phase II study was oxaliplatin $85 \mathrm{mg} / \mathrm{m}^{2}$ on day 1 and 29 plus capecitabine $1000 \mathrm{mg} / \mathrm{m}^{2}$ twice daily during 14 days starting on day 1 and 25 in combination with radiotherapy at a total dose of $50.4 \mathrm{~Gy}$. In our phase I/II study, we found grade III diarrhea in $19 \%$, a $\mathrm{R} 0$ resection in $81 \%$, downstaging to $\mathrm{T} 0-2$ in $33 \%$, and a pCR in $10 \%$ of patients. Table 3 summarizes the studies with the addition of oxaliplatin to a fluoropyrimidine (IV or orally) and radiotherapy. These studies show a toxicity and efficacy profile comparable to the results from our study.

Diarrhea generally occurred during the last 2 weeks of chemoradiation, which was accompanied by a significant decrease in QOL. As shown in Table 2, the addition of oxaliplatin increases the incidence of toxicities when compared with the use of $5 \mathrm{FU}$ alone. The observed postsurgical morbidity (mild wound infections excluded), the reoperations and the median hospitalization time in our study were $30 \%, 15 \%$, and 14 days, respectively, and are comparable with other studies. $^{24-26}$

We found a similar efficacy in $\mathrm{pCR}$ and $\mathrm{R} 0$ compared to other studies (Table 3). However, comparing the efficacy of these regimes is hampered by a number of technicalities. These comprise differences in 1) staging techniques, 2) the determination of distance of the tumor to the endopelvic fascia, 3) pathology techniques, and 4) the difference in interval between neoadjuvant treatment and surgery. The staging techniques used to define the locally advanced stage of the primary tumor and lymph nodes are clinical examination, CT, MRI, endoultrasound or combinations, the use of ultrasmall particles of iron oxide (USPIOs). ${ }^{27}$ These different staging techniques may lead to variabilities in pretreatment staging and therefore compromise the quantitation of downstaging. Moreover, the tumor might be still visible on posttreatment imaging, but it may have become nonvital. The distance of the tumor to the endopelvic fascia will influence the local recurrence rate. The determination of a $\mathrm{pCR}$ and an $\mathrm{R} 0$ resection depends on the sophistication of the pathology techniques used, and the definition of a R0 resection (distance tumor to resection or to CRM) is not uniform. In the various clinical protocols, the time interval between chemoradiotherapy and surgery is not standardized, which may influence the degree of downstaging but also the ultimate results of local cure. Therefore, cross-study comparison should be interpreted with caution. The end points used in this study are known to have a different impact on clinical outcome: a pCR is associated with a low local recurrence rate, and prolonged DFS and is not significantly related to the
cT and cN categories. ${ }^{28} \mathrm{R} 0$ resection is known to be a highly relevant prognostic factor. ${ }^{29}$ Comparing our results on pCR and R0 to other oxaliplatin-containing studies, we found similar results; however, these were also observed using monotherapy with $5 \mathrm{FU}$ or capecitabine (Table 2). Therefore, it is yet unknown whether the increased toxicity of adding oxaliplatin to a fluoropyrimidine in neoadjuvant chemoradiation schedules results in a clinical benefit for patients with rectal cancer.

In conclusion, this multicenter study demonstrated a neoadjuvant regimen with Capox-RT with an acceptable acute toxicity profile. Randomized phase III studies with in the standard arm 5FU will be necessary to show the true benefit of this approach. In such prospective studies, the standardization of the staging technique (i.e., the distance of the tumor to the endopelvic fascia), TME surgery, radiotherapy, and pathology should minimize the influence of these factors on the outcome.

\section{ACKNOWLEDGMENT}

There are no conflicts of interest. We thank the Dutch Integrale Kankercentra (IKC) for data management.

\section{REFERENCES}

1. Visser O, Siesling S, van Dijk JAAM. Incidence of cancer in the Netherlands 1999/2000: eleventh report of the National Cancer Registry. www.ikcnet.nl 2003.

2. Heald RJ, Ryall RD. Recurrence and survival after total mesorectal excision for rectal cancer. Lancet 1986; 1:1479-82.

3. McCall JL, Cox MR, Wattchow DA. Analysis of local recurrence rates after surgery alone for rectal cancer. Int $J$ Colorectal Dis $1995 ; 10: 126-32$.

4. Kapiteijn E, Marijnen CAM, Colenbrander AC, et al. Local recurrence in patients with rectal cancer diagnosed between 1988 and 1992: a population-based study in the west Netherlands. Eur J Surg Oncol 1998; 24:528-35.

5. Carlsen E, Schlichting E, Guldvog I, Johnson E, Heald RJ. Effect of the introduction of total mesorectal excision for the treatment of rectal cancer. Br J Surg 1998; 85:526-9.

6. Martling AL, Holm T, Rutqvist LE, Moran BJ, Heald RJ, Cedemark B. Effect of a surgical training programme on outcome of rectal cancer in the County of Stockholm. Stockholm Colorectal Cancer Study Group, Basingstoke Bowel Cancer Research Project. Lancet 2000; 356:93-6.

7. Adam IJ, Mohamdee MO, Martin IG, Scott N, Finan PJ, Johnston D, Dixon MF, Quirke P. Role of circumferential margin involvement in the local recurrence of rectal cancer. Lancet 1994; 344:707-11.

8. Marijnen CAM, Nagtegaal ID, Klein Kranenbarg E, Hermans J, van de Velde CJ, Leer JW, van Krieken JH. Pathology Review Committee and the Cooperative Clinical Investigators. No downstaging after short-term preoperative radiotherapy in rectal cancer. J Clin Oncol 2001; 19:1976-84. 
9. Bujko K, Nowacki MP, Nasierosska- Guttmejer A, et al. Sphincter preservation following preoperative radiotherapy for rectal cancer: report of a randomised trial comparing shortterm radiotherapy vs. conventionally fractionated radiochemotherapy. Radiother Oncol 2004; 72:15-24.

10. Reerink O, Verschueren RC, Szabo BG, Hospers GA, Mulder NH. A favourable pathological stage after neoadjuvant radiochemotherapy in patients with initially irresectable rectal cancer correlates with a favourable prognosis. Eur J Cancer 2003; 39:192-5.

11. Bosset JF, Collette L, Calais G, et al. Chemotherapy with preoperative radiotherapy in rectal cancer. New Engl Med 2006; 355:1114-23.

12. Bosset JF, Calais G, Mineur L, et al. Enhanced tumorocidal effect of chemotherapy with preoperative radiotherapy for rectal cancer: preliminary results-EORTC 22921. J Clin Oncol 2005; 23:5620-7.

13. Gerard JP, Conroy T, Bonnetain F, et al. Preoperative radiotherapy with or without concurrent fluorouracil and leucovorin in T3-4 rectal cancers: results of FFCD 9203. J Clin Oncol 2006; $24: 4620-5$

14. O'Connell MJ, Martenson JA, Wieand HS, et al. Improving adjuvant therapy for rectal cancer by combining protractedinfusion fluorouracil with radiation therapy after curative surgery. New Engl J Med 1994; 331:502-7.

15. de Gramont A, Bosset JF, Milan C, et al. Randomized trial comparing monthly low-dose leucovorin and fluorouracil bolus with bimonthly high-dose leucovorin and fluorouracil bolus plus continuous infusion for advanced colorectal cancer: a French intergroup. study, J Clin Oncol 1997; 15:808-15.

16. Yerushalmi R, Idelevich E, Dror $\mathrm{Y}$, et al. Preoperative chemoradioradiation in rectal cancer: retrpsepctive comparison between capecitabine and contiuous infusion of 5-fluorouracil. J Clin Oncol 2006; 93:529-33.

17. Punt CJA. New options and old dilemmas in the treatment of patients with advanced colorectal cancer. Ann Oncol 2004; 15:1453-9.

18. Magné N, Fischel JL, Formento P, et al. Oxaliplatin-5-fluorouracil and ionizing radiation. Importance of the sequence and influence of p53 status. Oncology 2003; 64:280-7.

19. Cividalli A, Ceciarelli F, Livdi E, Altavista P, Cruciani G, Marchetti P, Danesi DT. Radiosensitization by oxaliplatin in a mouse adenocarcinoma: influence of treatment schedule. Int $J$ Radiat Oncol Biol Phys 2002; 52:1092-8.

20. Khushalani NI, Leichman CG, Proulx G, et al. Oxaliplatin in combination with protracted-infusion fluorouracil and radiation: report of a clinical trial for patients with esophageal cancer. J Clin Oncol 2002; 20:2844-50

21. Enker WE, Thaler HT, Cranor ML, Polyak T. Total mesorectal excision in the operative treatment of carcinoma of the rectum. J Am Coll Surg 1995; 181:335-46.

22. Spitzer WO, Dobson AJ, Hall J, et al. Measuring the quality of life of cancer patients: a concise QL-index for use by physicians. J Chron Dis 1981; 34:585-97.

23. Bosset JF, Calais G, Daban A, et al. Preoperative chemoradiotherapy versus preoperative radiotherapy in rectal cancer patients: assessment of acute toxicity and treatment compliance. Report of the 22921 randomised trial conducted by the EORTC Radiotherapy Group. Eur J Cancer 2004; 40:219-24.

24. Machiels JP, Duck LR, Honhon B, Coster B, Scalliet P, Aydin S.. Phase II study of preoperative oxaliplatin, capecitabine, and external beam radiotherapy in patients with locally advanced rectal adenocarcinoma: the RadiOxCape study. Ann Oncol 2005; 16:1898-1905.

25. Aschele C, Friso ML, Pucciarelli S, et al. A phase I-II study of weekly oxaliplatin, 5-fiuorouracil continuous infusion and preoperative radiotherapy in locally advanced rectal cancer. Ann Oncol 2005; 16:1140-6.

26. Gerard JP, Chapet O, Nemoz C, et al. Preoperative concurrent chemoradiotherapy in locally advanced rectal cancer with high-dose radiation and oxaliplatin-containing regimen: the Lyon R0-04 phase II trial. J Clin Oncol 2003; 21:1119-24.

27. Koh DM, Brown G, Temple L, et al. Rectal cancer: mesorectal lymph nodes at MR imaging with USPIO versus histopathologic findings - initial observations. Radiology 2004; 231:91-9.

28. Rodel C, Martus P, Papadoupolos T, et al. Prognostic significance of tumor progression after preoperative chemoradiotherapy for rectal cancer. J Clin Oncol 2005; 34:8688-96.

29. Mawdsley S, Glynne-Jones R, Grainger J, et al. Can histopathologic assessment of circumferential margin after preoperative pelvic chemoradiotherapy for T3-T4 rectal cancer predict for 3-year disease-free-survival?. Int J Radiat Oncol Biol Phys 2005; 63:745-52.

30. Kim JS, Kim JS, Cho MJ, Song KS, Yoon WH. Preoperative chemoradiation using oral capecitabine in locally advanced rectal cancer. Int J Radiat Oncol Biol Phys 2002; 54:403-8.

31. Dunst J, Reese T, Debus J, et al. Phase-II-study of preoperative chemoradiation with capecitabine in rectal cancer. J Clin Oncol 2004; 22s:3559.

32. Glynne-Jones R, Sebag-Montefiore D, Maughan TS, Falk SJ, McDonald AC. A phase I dose escalation study of continuous oral capecitabine in combination with oxaliplatin and pelvic radiation (XELOX-RT) in patients with locally advanced rectal cancer. Ann Oncol 2006; 17:50-6.

33. Chau I, Brown G, Cunningham D, et al. Neoadjuvant capecitabine and oxaliplatin followed by synchronous chemoradiation and total mesorectal excision for MRI defined poor-risk rectal cancer. J Clin Oncol 2006; 24:668-74.

34. Gambacorta MA, Valentini V, Coco C, et al. Chemoradiation with raltitrexed and oxaliplatin in preoperative treatment of stage II-III resectable rectal cancer: Phase I and II studies. Int $J$ Radiat Oncol Biol Phys 2004; 60:139-48.

35. Rodel C, Grabenbauer G, Papadopoulos T, Hohenberger W, Schmoll HJ, Sauer R. Phase I/II trial of capecitabine, oxaliplatin and radiation for rectal cancer. J Clin Oncol 2003; 21:3098-104.

36. Carraro S, Roca EL, Cartelli C, et al. Radiochemotherapy with short daily infusion of low-dose oxaliplatin, leucovorin, and 5FU in T3-T4 unresectable rectal cancer: a phase II IATTGI study. Int J Radiat Oncol Biol Phys 2002; 54:397-402. 